\title{
Spying an invisible Higgs
}

\author{
Catherine Bernaciak ${ }^{1}$, Tilman Plehn ${ }^{1}$, Peter Schichtel $^{1,2}$, and Jamie Tattersall ${ }^{1}$ \\ 1 Institut für Theoretische Physik, Universität Heidelberg, Germany and \\ 2 Institute for Particle Physics Phenomenology, \\ Department of Physics, Durham University, UK
}

We investigate the potential of multivariate techniques to improve the LHC search for invisible Higgs decays in weak boson fusion. We find that in the coming runs the LHC will be able to probe an invisible Higgs width of $28 \%$ within a year and $3.5 \%$ during a high luminosity run. A significant improvement over these estimates requires an analysis of QCD radiation patterns down to $10 \mathrm{GeV}$. Such an analysis can improve the reach at the high luminosity run to $2 \%$. Throughout our analysis we employ a conservative, data driven background determination. 


\section{CONTENTS}

I. Introduction

II. Signal kinematics 3

III. Backgrounds 5

IV. LHC Reach 6 6

V. Conclusions 9

References 9 


\section{INTRODUCTION}

The discovery of the Higgs boson [1] on 4th July 2012 was a triumph for the LHC physics program [2. After the one missing piece of the Standard Model (SM) was found, attention quickly turned to measuring the properties of the particle. So far, all measurements at the LHC appear to be compatible with the Standard Model. Most notably, this holds for the measurement of the Higgs couplings to all Standard Model particles [3]. Given the multitude of indirect constraints on the Higgs sector and the sizable error bars this is not completely unexpected. With an upgrade in energy and much more data to collect, it is vital that all of the possible production and decay modes of the Higgs are probed to as high accuracy as possible.

The question of whether the Higgs boson has an invisible decay width is particularly important. In the Standard Model, such an invisible width is negligible compared to the expected LHC reach. However, many theories of new physics predict an invisible decay width competitive with the total Standard Model width 4 . The main motivation for searching for such decays is that the Higgs sector could be linked to a solution to dark matter. More precisely, due to the super-renormalizable nature of the Higgs mass term, any singlet field can mix with the Higgs and a portal into a hidden sector opens [5]. The Higgs portal opens a wealth of options for model building ranging from simple dark matter models to more complicated unified models [5]. Whatever guides the exact composition of such a hidden sector, a Higgs portal would always show itself through an invisible decay width [6], which sometimes leads to somewhat misguided speculation [7. A measurement of an invisible Higgs decay would therefore directly lead to interesting physics beyond the Standard Model and hopefully be connected to a viable dark matter candidate.

Recently hints of new physics have appeared in final states with missing energy at the LHC $\left[8\right.$ in the $W^{+} W^{-}$ cross-section and supersymmetric electroweak searches. All these anomalies involve missing energy in final states connected to electroweak symmetry breaking, which means they motivate improvements to the current invisible Higgs searches.

There are different strategies to detect such a deviation at the LHC. The classic search strategy for invisible Higgs decays is based on weak-boson-fusion (WBF) Higgs production [9, 10. Boosted Higgs production in association with a $W$ and $Z$ boson will significantly add to the LHC reach [11. A search for invisible Higgs decays in $t \bar{t} H$ production [12] will be a challenge at the LHC, both statistically and systematically. Experimental measurements in $Z H$ production and weak-boson-fusion Higgs production have recently been performed by CMS 13 and for $Z H$ production only by ATLAS [14. For a given underlying model, global fits of the Higgs couplings can probe invisible decay modes contributing to the total Higgs width in a model-dependent fashion [15 17.

In this study we analyze the WBF production channel and ask the question where it can be improved over the original findings [9]. We use a multivariate analysis implemented as a boosted decision tree (BDT) to separate signal and background and compare it to a normal cut based approach. One of the central questions is how much information is lost when we employ a jet veto instead of a comprehensive study of the jet activity. In addition, we devise a completely data driven background determination with essentially no Monte-Carlo extrapolation between control and signal regions. Specifically, the $Z \rightarrow \nu \nu$ background is modeled from $Z \rightarrow \ell \ell$ events and the $W \rightarrow \ell \nu$ background is determined from events with a lepton and a transverse mass consistent with a $W$-decay. The remaining systematic uncertainty is associated with lepton reconstruction and identification probabilities and the $W$ and $Z$ branching ratios. This means that the BDT can be trained on data and then applied to the signal region, which allows us to safely push the multivariate analysis without worrying about the effect this may have on the associated systematic error of the analysis.

In Section $[\mathrm{I}$ we start by explaining the kinematic variables entering the multivariate analysis, both with two tagging jets only and including additional QCD jets. In the following Section [II we present our background determination. Based on those two chapters we systematically test the prospects during the upcoming LHC runs in Section IV] We estimate the effectiveness of a central jet veto and find that the most promising path for an improvement of the WBF analysis is to include more information on QCD jet radiation.

\section{SIGNAL KINEMATICS}

All signal and background samples were produced by SHERPA 18 with additional matrix element jets matched using the CKKW algorithm [19]. The events are then passed through the DeLPHES detector simulation [20] 
with advanced parameterizations of the ATLAS electron, muon and tau reconstruction and identification algorithms given by the CheckMate tune [21. The final state jets are clustered using the anti- $k_{T}$ algorithm in FASTJET 22 with $R=0.4$.

To effectively separate signal and backgrounds we use a Boosted Decision Tree (BDT) available through TMva 23 in the Root analysis framework 24]. We use a BDT with 400 trees which each contain 3 layers and extensively test with different Monte-Carlo parameters to check that the trees are stable and not over-trained. To calculate the reach of the LHC we use the CLs prescription [25] and display our results in terms of the invisible Higgs width that can be excluded at the $95 \%$ confidence level.

All signal events are first required to pass a common set of trigger and selection cuts, including at least two tagging jets and missing transverse energy with

$$
\begin{aligned}
p_{T, j} & >20(10) \mathrm{GeV} & \left|\eta_{j}\right| & <4.5 \\
p_{T} & >100 \mathrm{GeV} & \Delta \phi_{p_{T}, j} & >0.4 .
\end{aligned}
$$

The angular separation of the jets and the missing energy vector helps to reduce fake missing energy from mis-measured jet momenta. For the signal selection we veto leptons, as described in the following Sec. III] Events including leptons from $W$ or $Z$ decays will correspondingly serve as control regions. We note that while the two tagging jets may appear too soft to be used as a trigger, we still require substantial missing energy, $p_{T}>100 \mathrm{GeV}$. Consequently, one or both jets will by definition have significant $p_{T}$ that can be triggered on.

In addition to conservatively using jets with $p_{T}>20 \mathrm{GeV}$ we also present results with $10 \mathrm{GeV}$ jets. This allows us to estimate possible improvements from the jet kinematics or a central jet veto [26]. However, there are significant doubts of how well this technique will work once pile-up is included. On the other hand, particle flow has shown significant promise in managing the effects of pile-up. This can especially be seen in boosted jet studies. On the theory side the number and the kinematic features of $10 \mathrm{GeV}$ jets are challenging to predict and will induce large errors in the analysis. Concerning the experimental systematics and the theory uncertainty we emphasize that our backgrounds are determined in a completely data driven way, as described in Sec. III. Any source of soft or collinear QCD radiation will equally affect both the background control regions and signal regions.

The first set of variables we define are the usual variables used in many WBF analyses along with $p_{T}$ due to the invisible Higgs signal we are searching for,

$$
\left\{p_{T, j},\left|\eta_{j_{1}}-\eta_{j_{2}}\right|, \eta_{j_{1}} \cdot \eta_{j_{2}}, m_{j_{1} j_{2}}, \Delta \phi_{j_{1}, j_{2}}, p_{T}\right\} \quad \text { (default) . }
$$

From the original analysis of the LHC sensitivity to an invisible Higgs [9] we quote the cut values with the exception of a the maximum jet rapidity,

$$
\begin{aligned}
& p_{T, j}>40 \mathrm{GeV} \\
& \not p_{T}>100 \mathrm{GeV} \\
& \left|\eta_{j}\right|<4.5 \\
& \left|\eta_{j_{1}}-\eta_{j_{2}}\right|>4.4 \\
& m_{j_{1} j_{2}}>1200 \mathrm{GeV} \\
& \eta_{j_{1}} \cdot \eta_{j_{2}}<0 .
\end{aligned}
$$

To easily compare the various results we always present the signal to background ratio after these default cuts. The central jet veto is defined by vetoing events with a third central jet $\eta_{j_{1}}>\eta_{j_{3}}>\eta_{j_{2}}$ where $p_{T, j_{3}}>20 \mathrm{GeV}[26$. For the box cuts defined above we order the jets in terms of decreasing $p_{T}$ to easily compare and check our results against the original study.

Finally, the azimuthal angle between the tagging jets with its peculiar sensitivity to the Lorentz structure of the event [9, 27] is added,

$$
\Delta \phi_{j_{1}, j_{2}}<1
$$

and we include these cuts in turn to give three baseline comparison points.

In a systematic analysis of the multi-jet kinematics we can in principle rely on Fox-Wolfram moments 28 . However, the invisible Higgs analysis is dominated by the 2-jet sample with a central jet veto, which can be easily described in terms of a few tagging jet observables. We therefore use a BDT including simply the kinematic variables

$$
\left\{p_{T, j_{1}}, \eta_{j_{1}}, p_{T, j_{2}}, \eta_{j_{2}}, \Delta \phi_{j_{1}, j_{2}}, \not p_{T}\right\} \quad(2 \text {-jet })
$$


While the actual kinematic variables differ between our 2-jet setup and the default set in Eq.22), we have checked that in a multivariate analysis the two sets are equivalent. When looking at the 3 -jet system we add

$$
\left\{p_{T, j_{3}}, \eta_{j_{3}}, \Delta \phi_{j_{1}, j_{3}}\right\} \quad \text { (3-jet) . }
$$

For this set of variables we use a forward-backward selection where we define $\eta_{j_{1}}=\eta_{\max }$ (the most forward jet) and $\eta_{j_{2}}=\eta_{\min }$ (the most backward jet). Any additional jets in the event are then ordered in terms of decreasing $p_{T}$. Consequently, with this selection, the events after a central jet veto and the exclusive 2-jet sample are identical. We have checked that the forward-backward selection of the tagging jets gives a better reach for the analysis than using a $p_{T}$ based selection.

At the matrix element level, the above variables without the missing transverse energy fully describe the system. However, additional soft radiation will always be present and produce a transverse boost to the system. This is why we add $p_{T}$ to the set of variables which we will target with the help of a multivariate analysis setup. In Sec [V] we will give a detailed argument why we can limit ourselves to the 2-jet and 3-jet samples described above.

\section{BACKGROUNDS}

The background to the invisible Higgs signal is dominated by two sources, $Z \rightarrow \nu \nu$ and $W \rightarrow \ell \nu$ where the final state lepton is either outside the detector acceptance or fails to be reconstructed. The $W$ and $Z$ production process in association with $n$ jets can be mediated by a pure QCD process radiating the weak gauge boson, $\sigma \propto \alpha_{s}^{n} \alpha$. Alternatively, there can be an underlying weak process with jet radiation, $\sigma \propto \alpha_{s}^{n-2} \alpha^{3}$. The QCD process will have a significantly larger rate, while the kinematics of the jet radiation will be more signal-like for the weak production process 26 . Both QCD multi-jet and $t \bar{t}$ production were found to contribute a negligible background compared to $W$ and $Z$ production. In particular the multi-jet background where one jet is highly mis-measured leading to a large $\not_{T}$ signal is very effectively reduced by the $\Delta \phi_{\phi_{T}, j}$ cut.

Our signal events have to pass a lepton veto, implemented in CheckMate 21]. The electron reconstruction uses the ATLAS 'loose' working point and is parametrized as a function of $p_{T}$ and $\eta$. The tau veto also relies on the ATLAS 'loose' working point as a function of $p_{T}$ and has roughly $70 \%$ efficiency for 1-prong and $65 \%$ efficiency for 3-prong hadronic tau decays. Both the $Z \rightarrow \nu \nu$ background and $W \rightarrow \ell \nu$ background where the lepton either fails to be reconstructed or falls outside of the detector acceptance are determined through control regions. To determine the backgrounds we rely completely on events with a reconstructed leptonic $Z$ or $W$ decay either after cuts or in a BDT trained with signal and background events. In both cases the backgrounds can be understood in detail using real events. We demand that these control region events pass exactly the same cuts as for the signal events. Leptons are required to fulfill

$$
\begin{array}{ll}
p_{T, e}>10 \mathrm{GeV} & \left|\eta_{e}\right|<2.5 \\
p_{T, \mu}>5 \mathrm{GeV} & \left|\eta_{\mu}\right|<2.5 \\
p_{T, \tau}>20 \mathrm{GeV} & \left|\eta_{\tau}\right|<2.5 .
\end{array}
$$

For the $W$ background, we require an isolated muon or electron instead of a lepton veto. In addition, the transverse mass of the lepton and the missing transverse momentum has to reconstruct the $W$-mass with $30 \mathrm{GeV}<m_{T}<100 \mathrm{GeV}$. For the $Z$ control region we require a pair of leptons of same flavor but opposite charge instead of the lepton veto. Their invariant mass has to reconstruct the $Z$-mass, $66 \mathrm{GeV}<m_{\ell \ell}<116 \mathrm{GeV}$.

Previous studies 9, 10 reduce the statistical uncertainty on the backgrounds by kinematically extrapolating from control regions with large background populations to signal regions with far smaller backgrounds. Essentially, the Monte-Carlo prediction is normalized to data in the control region and it is assumed that the shape is described well enough to extrapolate to the signal region with far smaller background. However, we find that the systematic uncertainty associated with such extrapolations can easily become the dominant error source at the LHC, especially for the higher luminosity runs. In addition, reliably estimating the size of this extrapolation uncertainty accurately will be a challenge once we have to decide if a measurement actually points to invisible Higgs decays.

Another approach to estimate the $Z \rightarrow \nu \nu$ background has been pioneered by the mono-jet searches at the LHC. It uses a single hard photon as a template for the $Z$. Again the motivation to include these events is the 


\begin{tabular}{r|cccc|cr}
\hline & \multicolumn{4}{|c|}{$p_{T, j}>20 \mathrm{GeV}$} & \multicolumn{2}{c}{$p_{T, j}>10 \mathrm{GeV}$} \\
\hline $\mathcal{L}\left[\mathrm{fb}^{-1}\right]$ & Eq. $\sqrt{3}$ & + jet veto & $+\Delta \phi_{j j}$ & BDT 2-jets & BDT 2-jets & 0.18 \\
10 & 1.02 & 0.49 & 0.47 & 0.28 & 0.07 & 0.061 \\
100 & 0.49 & 0.20 & 0.18 & 0.10 & 0.025 & 0.021 \\
3000 & 0.25 & 0.094 & 0.069 & 0.035 & BDT 3-jets \\
\hline
\end{tabular}

Table I. Exclusion reach in $\mathrm{BR}_{\text {inv }}=\Gamma_{\text {inv }} / \Gamma_{H}$ at $95 \%$ CLs to an invisible Higgs width at various luminosities and different combinations of cuts and multivariate analyses.

larger corresponding cross-section that reduces the associated statistical error. However, this also comes at the cost of introducing a non-trivial systematic uncertainty to account for the different kinematic structures. The limited systematic control can be studied in the distribution of the number of jets radiated in hard photon and $Z$ events [29].

We aim to minimize such extrapolations as much as possible and demand that the control samples with reconstructed leptons pass the same cuts as for the signal. In fact, the only extrapolation in our study are the cases when either a neutrino in $Z \rightarrow \nu \nu$ or a lepton in $W \rightarrow \ell \nu$ lies outside the detector acceptance. These events are rare since they are associated with small $p_{T}$, and the Monte-Carlo extrapolation of the $W$ and $Z$ decays will be very accurate.

The advantage of using a purely data driven background determination technique with minimal extrapolation is that it offers the perfect test bed to push multi-variate techniques in the search for new physics. Since the backgrounds are safely determined from data there is no danger of over-training on a Monte-Carlo effect that is not present in reality. We essentially use the background with reconstructed leptons as a template for the zero-lepton background. Deviations in the signal data from the background template give you access to the new physics. The translation between the two relies on the ratio,

$$
R=\frac{\operatorname{BR}(Z \rightarrow \nu \nu)}{\operatorname{BR}(Z \rightarrow \ell \ell)} \simeq 2.97
$$

for $\ell=e, \mu$. Currently, there is a $2 \%$ uncertainty on the ratio [30] and this is unlikely to improve in the LHC lifetime.

In addition, there are systematic uncertainties associated with the lepton identification, reconstruction and acceptance on both the background and control regions. Here we use the current CMS mono-jet search [31. as guide since the background is estimated in a very similar way. Currently the systematic uncertainty ranges around $3 \%$ for the $Z \rightarrow \nu \nu$ background and around $4 \%$ for the $W \rightarrow \ell \nu$ background. However, this error is dominated by the statistical error associated with the number of leptons reconstructed at different energies and angles. We scale this systematic error by the luminosity for each of the scenarios that we consider.

We would also like to note that the average $p_{T}$ for $Z \rightarrow \nu \nu$ background events after our BDT has been applied is $\sim 200 \mathrm{GeV}$ for all values of the discriminator used in our analysis. Consequently, we do not require the reconstruction of high energy leptons that may have larger uncertainties attached.

\section{LHC REACH}

To compute the prospects of the upcoming LHC runs at $13 \mathrm{TeV}$ in searching for an invisible Higgs produced in weak boson fusion we start with jets having $p_{T, j}>20 \mathrm{GeV}$. To reproduce the original analysis of Ref. 9] we apply the box cuts given in Eq.(3), add a central jet veto, and finally include the cut on the azimuthal tagging jet correlation in Eq.(4). We show the corresponding LHC reach in the invisible Higgs branching ratio at $95 \%$ CLs for different integrated luminosities in the first three columns of Tab. [1 Unlike in the original paper we do not include the jet veto survival probability as an external factor, but use our multi-jet simulations to simulate the jet veto. One of the reasons is that one of the assumptions in the computation of such survival properties, namely a Poisson distribution in the number of radiated jets, is strictly speaking not correct for the signal and for the electroweak background [26]. Nevertheless, our signal and background rates after the box cuts are consistent with the original work 9 .

With $10 \mathrm{fb}^{-1}$ at $13 \mathrm{TeV}$ we find that with the classical weak-boson-fusion cuts we can probe an invisible 

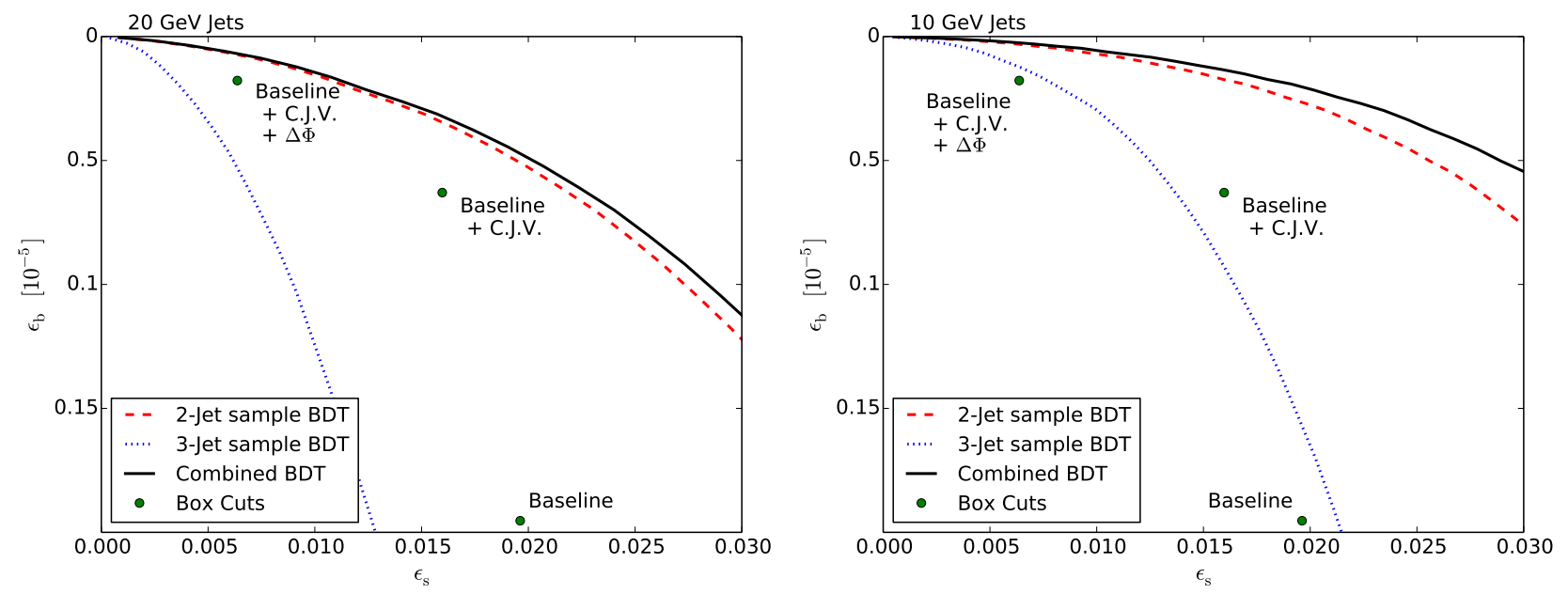

Figure 1. ROC curve for the 2-jet basis vectors, Eq. (5), and $\not_{T}$ on the exclusive $20 \mathrm{GeV}$ (left) and $10 \mathrm{GeV}$ (right) 2-jet sample (red dashed), 3-jet, also Eq. [6], (blue dotted) and the combination (black). For comparison the baseline box cut Eq. (3), also including a jet-veto and in addition a $\Delta \phi$ cut, Eq. (4) are shown as points.

width at the same order as the total Standard Model width. The jet veto is crucial to the analysis, increasing the reach in the invisible branching ratio by a factor two. Adding the $\Delta \phi_{j j}$ cut only marginally improves the limit at low luminosity. This is because with only $10 \mathrm{fb}^{-1}$ of data the analysis is statistically limited. Whilst the $\Delta \phi_{j j}$ cut improves the signal-to-background ratio, the significance is hardly affected. Our results for an integrated luminosity of $10 \mathrm{fb}^{-1}$ at $13 \mathrm{TeV}$ are similar to the CMS result (0.49) based on $20 \mathrm{fb}^{-1}$ at $8 \mathrm{TeV}$, which can be understood by the rough scaling of the number of events [13.

As we move to higher luminosity, the reduced statistical error allows us to probe smaller invisible Higgs widths. With $100 \mathrm{fb}^{-1}$ we expect to probe an invisible branching ratio around $20 \%$, while during the high-luminosity run with $3000 \mathrm{fb}^{-1}$ the limit should drop below $7 \%$. For the high-luminosity option the $\Delta \phi_{j j}$ box cut is at least as efficient as the central jet veto. This leads us to expect that in particular this cut will benefit from a multivariate analysis instead of a statistically limited cut applied to few remaining signal events.

To test the impact of a multi-variate analysis we start with a BDT that first only contains the 2-jet sample. The corresponding kinematic variables are listed in Eq.(5) and include the missing transverse energy because it is very specifically affected by soft physics. This approach can be viewed as a multi-variate analysis after a jet veto.

The power of a multi-variate analysis has to be described in terms of a receiver operator curve (ROC). On this curve we can eventually choose individual working points. The ROC curve of our BDT analysis is optimized to minimize the background for each chosen signal efficiency separately for the 2-jet and 3-jet samples. In the left panel of Fig. 1 we show the signal efficiency vs the background fake rate for the 2-jet sample compared to the three box cut results shown in Tab. I. For example assuming a constant signal efficiency we see that the BDT analysis halves the background fake rate, both compared to the basics cut with the central jet veto and the basic cuts with the central jet veto and the $\Delta \phi_{j j}$ cut.

The LHC reach of the multi-variate analysis is then calculated by including both the statistical and systematic uncertainty on the background. In fact, the systematic component of the background also has a statistical component in this analysis since we apply the same cuts on the background control samples as the background and signal itself. The ROC point with the most sensitivity for a particular luminosity is then chosen to calculate the final expected reach. The result for the 2-jet BDT analysis quoted in Tab. I suggests that the reach in the invisible branching ratio improves by a factor two for all luminosity scenarios. For the high-luminosity run an invisible branching ratio down to $3.5 \%$ can be probed.

The remaining key question is to what degree the full information on additional jets instead of the jet veto improves the LHC reach. For this purpose we train the BDT analysis on the combined 2-jet and 3-jet samples, using the extended set of kinematic variables given in Eq. (6). To see why we only consider the 2 -jet and 3 -jet sub-samples we examine the signal-to-background ratios in Tab. II For $20 \mathrm{GeV}$ jets, the original $S / B$ values 


\begin{tabular}{l|ccc|ccc}
\hline & \multicolumn{3}{|c|}{$p_{T, j}>20 \mathrm{GeV}$} & \multicolumn{3}{c}{$p_{T, j}>10 \mathrm{GeV}$} \\
\hline & 2 -jets & 3 -jets & 4 -jets & 2 -jets & 3 -jets & 4 -jets \\
\hline$S / B$ after Eq.11 & $1 / 240$ & $1 / 360$ & $1 / 475$ & $1 / 213$ & $1 / 303$ & 0.01 \\
$\epsilon_{S}$ & 0.01 & 0.01 & 0.01 & 0.01 & 0.01 \\
$\epsilon_{B}$ & $1.7 \times 10^{-6}$ & $1.3 \times 10^{-5}$ & $2.7 \times 10^{-5}$ & $7.5 \times 10^{-7}$ & $3.2 \times 10^{-6}$ & $2.4 \times 10^{-5}$ \\
$S / B$ & $1 / 2.6$ & $1 / 21$ & $1 / 42$ & $1 / 1.2$ & $1 / 5$ & $1 / 38$ \\
\hline
\end{tabular}

Table II. Signal and background efficiencies and signal-to-background ratios for different jet multiplicities after the acceptance cuts and after applying an optimized BDT discriminant. We choose the BDT discriminant such that $\epsilon_{S}=0.01$.

after the acceptance cuts of Eq.(1) decrease with the number of jets, leading to a statistical limitation of higher jet multiplicities. Because of the color structure of the signal the geometric distribution of the jets is very different for the signal and the background; this difference is most pronounced for the two tagging jets and gets washed out with any radiated jets. We test this by comparing the background efficiencies from the BDT for working point with a constant signal efficiency of $\epsilon_{S}=0.01$. This point is close to the optimal choice for an integrated luminosity of $10 \mathrm{fb}^{-1}$. In the corresponding $S / B$ values we indeed observe a dramatic drop for the 3-jet and 4-jet samples, compared to the same ratio after acceptance cuts only. If, as it will turn out, the 3-jet samples do not have significant impact on the final signal extraction we can safely neglect higher jet multiplicities.

In the left panel of Fig. 1 we see that for jets with $p_{T, j}>20 \mathrm{GeV}$ the performance of the 3 -jet analysis is marginal. This is not entirely unexpected. we know that only a small fraction of signal events has an additional jet, while the number of jets in QCD $Z$ +jets production is logarithmically enhanced and follows a Poisson distribution with a maximum at finite jet multiplicities $[26$. As a matter of fact, this observation has been the motivation for a central jet veto [26] and can be reproduced from Tab. II. Combining the 2-jet sample with the 3 -jet sample shows hardly any improvement. We only start to see a slight difference between the 2-jet sample and the full sample when we look at higher signal acceptances, which is not the regime which gives us the best reach for invisible Higgs decays for any of the three luminosity choices.

Using the same BDT setup as in Tab. II we can determine the composition of the different backgrounds to the 2 -jet sample. We find that the QCD $W+$ jets background is the largest with $\sigma_{B}=98 \mathrm{fb}$ for a signal cross section of $\sigma_{S}=80 \mathrm{fb}$. QCD $Z+$ jets is the second-largest background with $50 \mathrm{fb}$, followed by electroweak $Z+$ jets and $W+$ jets production with $37 \mathrm{fb}$ and $27 \mathrm{fb}$. This means that for our working point the QCD production process are slightly larger, but the electroweak processes are hardly suppressed.

The only obvious path to improve the LHC reach for invisible Higgs decays is to include more information on jet radiation in the analysis. First, a lower transverse momentum cut on the tagging jets will increase the signal statistics. Second, the structure of additional jet radiation will be more distinctive the more jets we include in the corresponding analysis. In Tab. I we compare the $10 \mathrm{GeV}$ and $20 \mathrm{GeV}$ jet selection for the 2-jet BDT analysis with the kinematic observables given in Eq.(5). Indeed, the experimentally challenging analysis setup including softer jets can increase the LHC reach in the invisible Higgs branching ratio by some $50 \%$.

In the right panel of Fig. 1 we compare the BDT with the box cuts using $10 \mathrm{GeV}$ jets, as before. In addition to the expected improvement in the 2-jet analysis we also observe a comparably dramatic effect on the 3 -jet analysis. While it is still not competitive with the 2-jet analysis, it leads to a significant improvement at large signal efficiencies of $\epsilon_{S}>2 \%$. For the optimal working point an improvement by almost a factor of 2 compared to the $20 \mathrm{GeV}$ dominantly 2 -jet analysis is shown in Tab. I]

In this case, where the 3-jet sample does allow for a significant improvement of the LHC reach we definitely have to see what additional jets can contribute. In Tab. [I] we again show the signal-to-background ratios for different jet multiplicities after acceptance cuts and after a BDT analysis of the kinematic features. While for the $20 \mathrm{GeV}$ case the dramatic loss of power occurred between the 2-jet and 3-jet samples, the softer jets above $p_{T, j}=10 \mathrm{GeV}$ move this drop to between the 3 -jet and the 4 -jet samples. This shift reflects the fact that for sufficiently low transverse momenta enough signal events will develop additional jet activity which is different from the corresponding background patterns. In this case the relevance of the different backgrounds get re-adjusted: while the dominant background remains QCD $W+$ jets production with $37 \mathrm{fb}$, it is now followed by electroweak $Z+$ jets production with $23 \mathrm{fb}$ and QCD $Z+$ jets production with $19 \mathrm{fb}$. The electroweak $W+$ jets channel adds $14 \mathrm{fb}$ to the combined backgrounds. Electroweak backgrounds exhibit a QCD structure very similar to the WBF signal, making them more dangerous the more we rely on jet patterns for the signal extraction. 


\section{CONCLUSIONS}

We have investigated how to improve the reach of the LHC to invisible Higgs decays in the classic weak-bosonfusion channel [9]. Based on a multi-variate BDT analysis we found that we can probe signal rates almost twice as small as with traditional cuts. In particular, at a $13 \mathrm{TeV}$ LHC with $10 \mathrm{fb}^{-1}$, we find that the reach improves from an invisible branching of $47 \%$ to $28 \%$ at $95 \%$ C.L. For the high-luminosity LHC we expect a final reach around $3.5 \%$, significantly benefiting from the increased statistics. Making use of large expected event samples in the coming LHC runs we completely rely on reconstructed $W$-and $Z$-decays for the background simulation, minimizing systematic and theoretical uncertainties.

The central question in our analysis is to what degree a central jet veto can be improved by taking into account the full information on the QCD jet radiation. For jets above $20 \mathrm{GeV}$ we find that the 3-jet configuration hardly contributes to the signal extraction unless we choose a working point with very large signal efficiencies. Correspondingly, the QCD $Z+$ jets and $W+$ jets backgrounds are slightly more dangerous than their electroweak counterparts.

The main improvement of invisible Higgs searches at the LHC in this channel needs to incorporate more information on the QCD activity in the signal and background events. This could be achieved by reducing the jet threshold to $10 \mathrm{GeV}$. While this is clearly not a conservative requirement on the detectors and the analysis strategy, methods to include soft jets are being tested for example in jet substructure studies. With the additional jets the 3-jet topology does contribute to the signal extraction and should be included beyond a central jet veto. The high-luminosity run will then be sensitive to branching ratios of $2.1 \%$, also limited by our understanding of the electroweak backgrounds.

\section{Acknowledgments}

We would like to thank Daniel Schmeier for the implementation of the CLs calculator used in this study. PS acknowledges support from the European Union as part of the FP7 Marie Curie Initial Training Network MCnet ITN (PITN-GA-2012-315877) and the IMPRS for Precision Tests of Fundamental Symmetries.

[1] P. W. Higgs, Phys.Lett. 12, 132 (1964); Phys.Rev.Lett. 13, 508 (1964) F. Englert and R. Brout, Phys.Rev.Lett. 13, 321 (1964) P. W. Higgs, Phys.Rev. 145, 1156 (1966).

[2] G. Aad et al. (ATLAS Collaboration), Phys.Lett. B716, 1 (2012), arXiv:1207.7214 [hep-ex]; S. Chatrchyan et al. (CMS Collaboration), Phys.Lett. B716, 30 (2012), arXiv:1207.7235 [hep-ex].

[3] M. Klute, R. Lafaye, T. Plehn, M. Rauch, and D. Zerwas, Phys.Rev.Lett. 109, 101801 (2012), arXiv:1205.2699 [hep-ph]; D. Lopez-Val, T. Plehn, and M. Rauch, JHEP 1310, 134 (2013), arXiv:1308.1979 [hep-ph]

[4] A. Djouadi, A. Falkowski, Y. Mambrini, and J. Quevillon, Eur.Phys.J. C73, 2455 (2013) arXiv:1205.3169 [hep-ph] C. Englert, A. Freitas, M. Mhlleitner, T. Plehn, M. Rauch, et al., J.Phys. G41, 113001 (2014), arXiv:1403.7191 [hep-ph]

[5] B. Patt and F. Wilczek, (2006), arXiv:hep-ph/0605188 [hep-ph]; M. Gonderinger, Y. Li, H. Patel, and M. J. RamseyMusolf, JHEP 1001, 053 (2010), arXiv:0910.3167 [hep-ph] O. Bertolami and R. Rosenfeld, Int.J.Mod.Phys. A23, 4817 (2008), arXiv:0708.1784 [hep-ph] S. Andreas, C. Arina, T. Hambye, F.-S. Ling, and M. H. Tytgat, Phys.Rev. D82, 043522 (2010), arXiv:1003.2595 [hep-ph]; C. Englert, J. Jaeckel, V. Khoze, and M. Spannowsky, JHEP 1304, 060 (2013), arXiv:1301.4224 [hep-ph].

[6] R. E. Shrock and M. Suzuki, Phys.Lett. B110, 250 (1982) L.-F. Li, Y. Liu, and L. Wolfenstein, Phys.Lett. B159, 45 (1985) K. Griest and H. E. Haber, Phys.Rev. D37, 719 (1988); I. Low, P. Schwaller, G. Shaughnessy, and C. E. Wagner, Phys.Rev. D85, 015009 (2012), arXiv:1110.4405 [hep-ph]; G. Belanger, B. Dumont, U. Ellwanger, J. Gunion, and S. Kraml, Phys.Lett. B723, 340 (2013), arXiv:1302.5694 [hep-ph]; M. Bento, O. Bertolami, and R. Rosenfeld, Phys.Lett. B518, 276 (2001), arXiv:hep-ph/0103340 [hep-ph]; C. Englert, T. Plehn, M. Rauch, D. Zerwas, and P. M. Zerwas, Phys.Lett. B707, 512 (2012), arXiv:1112.3007 [hep-ph]

[7] C. Englert, J. Jaeckel, E. Re, and M. Spannowsky, Phys.Rev. D85, 035008 (2012) arXiv:1111.1719 [hep-ph]

[8] D. Curtin, P. Jaiswal, and P. Meade, Phys.Rev. D87, 031701 (2013), arXiv:1206.6888 [hep-ph]; D. Curtin, P. Jaiswal, P. Meade, and P.-J. Tien, JHEP 1308, 068 (2013), arXiv:1304.7011 [hep-ph] K. Rolbiecki and K. Sakurai, JHEP 1309, 004 (2013), arXiv:1303.5696 [hep-ph] D. Curtin, P. Meade, and P.-J. Tien, (2014), arXiv:1406.0848 [hep-ph] J. S. Kim, K. Rolbiecki, K. Sakurai, and J. Tattersall, (2014), arXiv:1406.0858 [hep-ph]

[9] O. J. Eboli and D. Zeppenfeld, Phys.Lett. B495, 147 (2000), arXiv:hep-ph/0009158 [hep-ph]. 
[10] B. Di Girolamo and L. Neukermans, (2002); Y. Bai, P. Draper, and J. Shelton, JHEP 1207, 192 (2012) arXiv:1112.4496 [hep-ph]; D. Ghosh, R. Godbole, M. Guchait, K. Mohan, and D. Sengupta, Phys.Lett. B725, 344 (2013), arXiv:1211.7015 [hep-ph],

[11] D. Choudhury and D. Roy, Phys.Lett. B322, 368 (1994), arXiv:hep-ph/9312347 [hep-ph] R. Godbole, M. Guchait, K. Mazumdar, S. Moretti, and D. Roy, Phys.Lett. B571, 184 (2003) arXiv:hep-ph/0304137 [hep-ph]. H. Davoudiasl, T. Han, and H. E. Logan, Phys.Rev. D71, 115007 (2005) arXiv:hep-ph/0412269 [hep-ph] H. Okawa, J. Kunkle, and E. Lipeles, (2013), arXiv:1309.7925 [hep-ex].

[12] J. Gunion, Phys.Rev.Lett. 72, 199 (1994), arXiv:hep-ph/9309216 [hep-ph]; N. Zhou, Z. Khechadoorian, D. Whiteson, and T. M. Tait, (2014), arXiv:1408.0011 [hep-ph].

[13] S. Chatrchyan et al. (CMS Collaboration), Eur.Phys.J. C74, 2980 (2014), arXiv:1404.1344 [hep-ex]

[14] G. Aad et al. (ATLAS Collaboration), Phys.Rev.Lett. 112, 201802 (2014) arXiv:1402.3244 [hep-ex],

[15] R. Lafaye, T. Plehn, M. Rauch, D. Zerwas, and M. Duhrssen, JHEP 0908, 009 (2009), arXiv:0904.3866 [hep-ph].

[16] C. Englert, T. Plehn, D. Zerwas, and P. M. Zerwas, Phys.Lett. B703, 298 (2011), arXiv:1106.3097 [hep-ph]

[17] J. Espinosa, C. Grojean, M. Muhlleitner, and M. Trott, JHEP 1212, 045 (2012), arXiv:1207.1717 [hep-ph] J. R. Espinosa, M. Muhlleitner, C. Grojean, and M. Trott, JHEP 1209, 126 (2012) arXiv:1205.6790 [hep-ph]; P. P. Giardino, K. Kannike, M. Raidal, and A. Strumia, JHEP 1206, 117 (2012), arXiv:1203.4254 [hep-ph]; N. Desai, B. Mukhopadhyaya, and S. Niyogi, (2012), arXiv:1202.5190 [hep-ph]

[18] T. Gleisberg, S. Hoeche, F. Krauss, M. Schonherr, S. Schumann, et al., JHEP 0902, 007 (2009), arXiv:0811.4622 [hep-ph].

[19] S. Catani, F. Krauss, R. Kuhn, and B. Webber, JHEP 0111, 063 (2001), arXiv:hep-ph/0109231 [hep-ph]

[20] J. de Favereau, C. Delaere, P. Demin, A. Giammanco, V. Lematre, et al., (2013), arXiv:1307.6346 [hep-ex].

[21] M. Drees, H. Dreiner, D. Schmeier, J. Tattersall, and J. S. Kim, (2013), arXiv:1312.2591 [hep-ph]

[22] M. Cacciari and G. P. Salam, Phys.Lett. B641, 57 (2006), arXiv:hep-ph/0512210 [hep-ph]; M. Cacciari, G. P. Salam, and G. Soyez, JHEP 0804, 063 (2008), arXiv:0802.1189 [hep-ph]; Eur.Phys.J. C72, 1896 (2012), arXiv:1111.6097 [hep-ph].

[23] A. Hocker, J. Stelzer, F. Tegenfeldt, H. Voss, K. Voss, et al., PoS ACAT, 040 (2007), arXiv:physics/0703039 [PHYSICS]

[24] I. Antcheva, M. Ballintijn, B. Bellenot, M. Biskup, R. Brun, et al., Comput.Phys.Commun. 182, 1384 (2011).

[25] A. L. Read, J.Phys. G28, 2693 (2002)

[26] R. Kleiss and W. J. Stirling, Phys.Lett. B200, 193 (1988); U. Baur and E. N. Glover, Phys.Lett. B252, 683 (1990) V. D. Barger, K.-m. Cheung, T. Han, J. Ohnemus, and D. Zeppenfeld, Phys.Rev. D44, 1426 (1991); D. L. Rainwater, R. Szalapski, and D. Zeppenfeld, Phys.Rev. D54, 6680 (1996), arXiv:hep-ph/9605444 [hep-ph] B. E. Cox, J. R. Forshaw, and A. D. Pilkington, Phys.Lett. B696, 87 (2011), arXiv:1006.0986 [hep-ph]; E. Gerwick, T. Plehn, and S. Schumann, Phys.Rev.Lett. 108, 032003 (2012), arXiv:1108.3335 [hep-ph].

[27] T. Plehn, D. L. Rainwater, and D. Zeppenfeld, Phys.Rev.Lett. 88, 051801 (2002) arXiv:hep-ph/0105325 [hep-ph] V. Hankele, G. Klamke, D. Zeppenfeld, and T. Figy, Phys.Rev. D74, 095001 (2006), arXiv:hep-ph/0609075 [hep-ph] K. Hagiwara, Q. Li, and K. Mawatari, JHEP 0907, 101 (2009), arXiv:0905.4314 [hep-ph].

[28] C. Bernaciak, B. Mellado, T. Plehn, P. Schichtel, and X. Ruan, Phys.Rev. D89, 053006 (2014), arXiv:1311.5891 [hepph]; C. Bernaciak, M. S. A. Buschmann, A. Butter, and T. Plehn, Phys.Rev. D87, 073014 (2013), arXiv:1212.4436 [hep-ph].

[29] C. Englert, T. Plehn, P. Schichtel, and S. Schumann, JHEP 1202, 030 (2012), arXiv:1108.5473 [hep-ph].

[30] K. Olive et al. (Particle Data Group), Chin.Phys. C38, 090001 (2014)

[31] V. Khachatryan et al. (CMS Collaboration), (2014), arXiv:1408.3583 [hep-ex] 